\title{
Síndrome do bloqueio capsular precoce relato de caso e especulação sobre o tamanho da parte óptica da lente como fator de risco
}

\section{Early caspsular block syndrome a case report and speculation about the size of the lens' optic as a risk factor}

Eduardo Ferrari Marback ${ }^{1}$, Rita de Cássia Aquino de Araújo ${ }^{2}$, Marcelo Siqueira de Freitas ${ }^{3}$

\section{Resumo}

Apresentamos um caso de síndrome de bloqueio capsular precoce, tratado por reintervenção cirúrgica no segundo dia de pós-operatório. Comentamos as principais características da síndrome, os possíveis fatores predisponentes, as opções de tratamento e a importância do seu correto reconhecimento. Nós também especulamos sobre outro possível fator de risco, lentes intraoculares com parte óptica de $6 \mathrm{~mm}$ ou maiores.

Descritores: Cápsula do cristalino; Cristalino/cirurgia; Extração da catarata/efeitos adversos; Pseudofacia/complicações; Relatos de casos

\footnotetext{
${ }^{1}$ Doutor, Oftalmologista do Instituto de Olhos Freitas - Salvador (BA), Brasil;

${ }^{2}$ Oftalmologista do Instituto de Olhos Freitas - Salvador (BA), Brasil;

${ }^{3}$ Mestre, Diretor Médico do Instituto de Olhos Freitas - Salvador (BA), Brasil.
} 


\section{INTRODUÇÃO}

A síndrome do bloqueio capsular é uma complicação da cirurgia de catarata que surgiu após a introdução da capsulorrexe ${ }^{(1)}$.Pode ocorrer no per-operatório, pós-operatório precoce ou tardio, com múltiplas etiologias propostas ${ }^{(2,3)}$. Na forma per-operatória o núcleo do cristalino bloqueia a capsulorrexe durante a manobra de hidrodissecção, podendo levar a ruptura da cápsula posterior ${ }^{(2)}$. Nas formas pós-operatórias, tanto na precoce como na tardia é possível o bloqueio se dá através do contato entre a parte óptica da lente intraocular (LIO) e a capsulorrexe, sendo possível identificar acúmulo de líquido entre estas, o que desloca a LIO anteriormente gerando desvio miópico, podendo ainda diminuir a profundidade da câmara anterior e levar a aumento da pressão intraocular ${ }^{(1-5)}$.

Descrevemos aqui um caso de bloqueio caspular precoce tratado por nova intervenção cirúrgica no segundo dia de pós-operatório (DPO), questionando sobre sua possível relação com o diâmetro da parte óptica da LIO.

\section{Relato do caso}

Paciente masculino, 81 anos, submetido à facectomia por catarata senil em olho direito, sem intercorrências, pela técnica de facoemulsificação. Durante a cirurgia duas substâncias viscoelásticas diferentes foram utilizadas, para os estágios iniciais até a confecção da capsulorrexe, já para as etapas de irrigação/ aspiração e implante da LIO foi utilizada a metilcelulose a 2\% (Coatel, Baush Lomb). A facoemulsificação foi feita pela técnica de múltipla divisão circunferencial ${ }^{(6)}$, utilizando a combinação de sulfato de condroitina a $4 \%$ e hialuronato de sódio a 1,65\%(Discovisc, Alcon). Após o implante da LIO, realizamos irrigação/aspiração automatizada dos restos de viscoelástico da câmara anterior com mobilização lateral da LIO e tentativa de irrigação de solução salina balanceada atrás da parte óptica. O comprimento axial do olho à biometria óptica foi de 23,57 mm. A LIO implantada no saco capsular foi de peça única em acrílico hidrofílico (C Flex Apheric, Rayner) com refração esférica pós-operatória estimada em aproximadamente $-0,50$ dioptrias.

No primeiro DPO o paciente se apresentou afirmando que, apesar da programação cirúrgica para resultar em boa visão não corrigida para longe, estava enxergando melhor de perto. A acuidade visual (AV) não corrigida era de 0,1 . Com correção de -2,50 dioptrias esféricas e $-0,50$ dioptrias cilíndricas a $120^{\circ}$ a $\mathrm{AV}$ chegava a 0,5. Havia mínimo edema de córnea peri-incisional e traços de células inflamatórias em câmara anterior. No segundo DPO a AV e refração permaneciam inalteradas. Ao exame notava-se LIO centrada, porém com a presença de separação nítida e ampla entre a parte óptica da LIO e cápsula posterior, melhor identificada após midríase farmacológica (Figura 1). A câmara anterior parecia ter profundidade normal e a pressão intraocular era igual a $11 \mathrm{mmHg}$. Diante do diagnóstico de síndrome do bloqueio capsular precoce, optamos por retornar o paciente à sala cirúrgica. Após preparo com anestesia tópica e colírio de idopovidona a $5 \%$ e campos cirúrgicos adesivos, a incisão principal foi aberta com uma espátula de íris e o bloqueio capsular desfeito por aspiração e mobilização da LIO com uma cânula de irrigação/aspiração manual (Figura 2A). Ao fim do procedimento foi possível identificar a presença de dobra da cápsula posterior logo atrás da parte óptica da LIO, indicando não existir mais espaço real entre elas (Figura 2B).

No primeiro DPO a AV sem correção era igual a 0,5 , chegando a 0,7 com - 0,50 dioptrias esféricas, havendo mínimo edema corneano. No $7^{\circ} \mathrm{DPO}$, a AV sem correção era de 0,7 , chegando a 0,9 com $-0,25$ dioptrias esféricas e $-0,50$ dioptrias cilíndricas a $145^{\circ}$.

\section{Discussão}

A síndrome de bloqueio capsular precoce é rara, embora não existam dados que permitam conclusão definitiva de sua incidência. Durak e colaboradores rela$\operatorname{tam} 13$ casos em 1140 cirurgias $(1,14 \%$ ) e Kim e colaboradores encontraram 8 casos em 1.100 cirurgias $(0,73 \%)^{(3,4)}$. São relatados como fatores de risco diâmetro axial maior que $25 \mathrm{~mm}$ e uso de LIO de acrílico hidrofílico com quatro pontos de suporte, embora a síndrome já tenha sido identificada em pacientes com praticamente todos os tipos de materiais e desenhos de LIOs disponíveis ${ }^{(1-5)}$. Outros fatores que pensamos ter possível influência são o tamanho da capsulorrexe, o tipo de substância viscoelástica utilizada e a rotina de aspiração desta após o implante da $\mathrm{LIO}^{(1-5,6)}$. Existem duas teorias principais para justificar o acúmulo de líquido e a distensão do saco capsular na forma precoce da síndrome. Davison propõe que o epitélio capsular e remanescentes corticais gerariam uma pressão oncótica responsável pelo acúmulo de fluido ${ }^{(1)}$. Já Masket, especula que substância viscoleástica retida atrás da LIO, especificamente o hialuronato de sódio, levaria também a um gradiente oncótico e acúmulo de líquido ${ }^{(7)}$. A suposta presença de material viscolástico já foi observada por outros autores ${ }^{(2-5)}$, e comprovada pela análise do líquido realizada por Sugiura e colaboradores ${ }^{(8)}$. Possivelmente os dois mecanismos podem coexistir, como no relato de Mardelli e Mehana ${ }^{(9)}$. Em nosso caso, utiliza- 

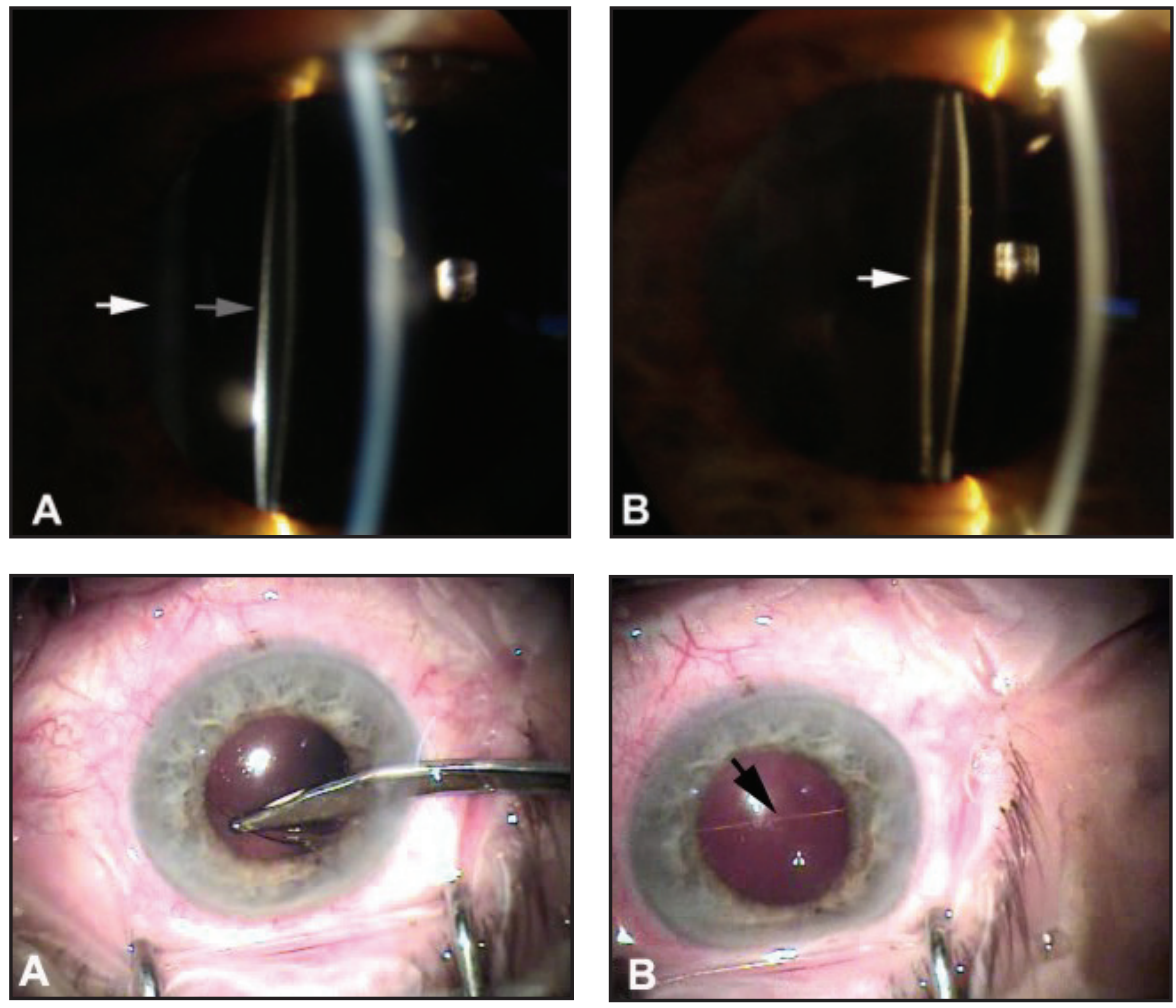

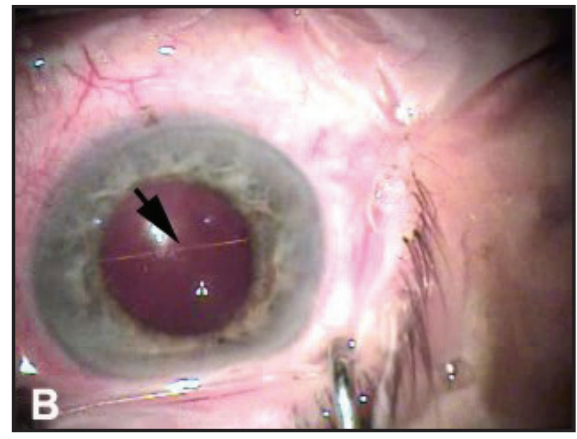

Figura 1: A) Aspecto biomicroscópico no segundo dia pós-operatório, espaço entre a parte óptica da lente intraocular (seta cinza) e cápsula posterior (seta branca); B) Sete dias pós irrigação/aspiração para desfazer o bloqueio capsular, nota-se parte óptica da lente intraocular praticamente em contacto direto com a cápsula posterior (seta)

Figura 2: A) Mobilizando a lente intraocular para desfazer o bloqueio; B) Notar dobra de cápsula posterior (seta) indicando não haver mais distensão do saco capsular mos dois viscoelásticos diferentes, um deles com hialuronato de sódio em sua composição.

A forma precoce da síndrome pode acontecer por bloqueio mecânico pela simples aposição da óptica da LIO à capsulorrexe, ou ainda por uma aderência inflamatória entre estas duas estruturas ${ }^{(3)}$. Em ambas situações costuma haver um deslocamento anterior da LIO com indução de desvio miópico que varia entre 2,0 a 3,0 dioptrias $^{(3-5)}$. Na forma inflamatória, o tratamento clínico da causa de base costuma resolver o bloqueio(3). Já a forma mecânica usualmente necessita de nova intervenção. Durak e colaboradores relatam resolução espontânea em apenas 2 de 13 olhos após observação por 30 dias, dos 11 olhos restantes em 8 foi tentada capsulotomia anterior por YAG laser, com resolução em 5 olhos, 5 foram tratados por capsulotomia posterior e 1 paciente optou por não tratar por sentir-se confortável com a miopia $^{(4)}$. Já Mardelli propõe o tratamento precoce, realizado por agulhamento na lâmpada de fenda ${ }^{(5)}$. Em nosso caso, optamos por levar novamente o paciente à sala de cirurgia e, após preparo de pele e saco conjuntival com iodopovidiona a $10 \%$ e $5 \%$ respectivamente, e correto isolamento de anexos com campo adesivo, abrimos a incisão principal e realizamos mobilização da LIO e aspiração do líquido por detrás da mesma, com uma cânula de irrigação/aspiração manual. No sétimo DPO a AV era de 0,9 e houve um desvio positivo de aproxima- damente 2,5 dioptrias, como relata a literatura ${ }^{(1-5)}$. Nossa opção de reintervenção cirúrgica foi tomada pela necessidade de restaurar a funcionalidade da visão de forma rápida e efetiva. Questionamos o uso da capsulotomia posterior por suas complicações potenciais como descolamento de retina e edema cistóide de macular, bem como pela necessidade de idealmente postergar a resolução do problema por no mínimo 30 dias de pósoperatório, já o agulhamento em lâmpada de fenda nos parece um procedimento potencialmente desconfortável para o paciente e cirurgião. Não sabemos qual o risco de endoftalmite ao reabrir a incisão principal da cirurgia em ambiente de centro cirúrgico mesmo com todo o preparo e cuidados referentes a uma cirurgia intraocular. Em nosso serviço nos últimos 67 meses realizamos 4520 facectomias e tivemos dois casos (0,044\%) de endoftalmite (dados não publicados), extrapolando esta taxa para a reabertura da incisão e irrigação/aspiração da câmara anterior, acreditamos que o risco de infecção é bastante pequeno e o conforto para o paciente e controle para o cirurgião são sem dúvida muito maiores.

Em nosso caso, a LIO utilizada foi de acrílico hidrofílico, em peça única, com alças de desenho duplo e parte óptica de $6,25 \mathrm{~mm}$. Embora este tenha sido o único caso identificado por nós em 106 cirurgias com esta LIO, é interessante notar que apesar de termos realizado ao redor de 4520 facectomias nos últimos 67 meses com a 
mesma técnica cirúrgica e LIOs de diferentes desenhos e materiais, ${ }^{(6)}$ este foi o primeiro caso diagnosticado por nós. Embora este dado não seja absoluto pois podemos ter tomado algum caso prévio como erro biométrico, achamos que isto é improvável. O acrílico hidrofílico já foi aventado como possível fator de risco para o bloqueio capsular precoce, embora existam casos relatados também com silicone, polimetilmetacrilato (PMMA) e acrílico hidrofóbico ${ }^{(2-4,7-9)}$. No trabalho de Kim e colaboradores foi relatado associação entre implante de LIO de acrílico hidrofílico e síndrome de bloqueio capsular, e em 206 implantes de LIOS Akreos Adapt (Baush Lomb) foram encontrados 6 casos (2,9\%) de síndrome do bloqueio capsular precoce ${ }^{(3)}$. Nossa experiência pessoal com este material ainda é limitada pois só começamos a utilizá-lo nos últimos 16 meses, neste período implantamos 498 lentes de peça única do mesmo acrílico hidrofílico com óptica de 5,75 mm (Akreos Fit, Baush Lomb), sem nunca ter visto um caso de bloqueio capsular. Vale ressaltar que apesar de serem do mesmo material, a Akreos adapt tem um desenho peculiar, com óptica de $6 \mathrm{~mm}$ e quatro alças que potencialmente limitam a mobilidade da LIO e a aspiração da substância viscoelástica atrás da LIO. Talvez mais importante que o material utilizado, seja a associação entre uma LIO de peça única sem angulação posterior de alças e, principalmente, uma parte óptica grande. Quando individualizamos as LIOs nas diversas séries publicadas, é nítido que a maioria dos casos aconteceram em olhos implantados com LIOs com pelo menos $6 \mathrm{~mm}$ de parte óptica ${ }^{(3-5)}$. Chamamos a atenção para a associação entre o implante de LIO com óptica grande e capsulorrexe pequena e bem centrada. Esta combinação tem se tornado cada vez mais frequente com a introdução das LIOs asféricas, tóricas e multifocais, que idealmente devem ficar no saco capsular com $360^{\circ}$ de parte óptica coberta pela borda da capsulorrexe para manter a LIO bem centrada, sendo nítida uma tendência à redução no diâmetro da capsulorrexe por grande parte dos cirurgiões.

É importante também chamar a atenção do cirurgião para o diagnóstico diferencial entre a síndrome de bloqueio capsular precoce e o erro biométrico. As soluções para estes dois problemas podem ser antagônicas. Ou seja, um caso de síndrome de bloqueio capsular tomado por erro biométrico pode levar a uma troca de LIO ou cirurgia refrativa corneana. A troca da LIO provavelmente resolveria o bloqueio, mas levaria a insatisfação refrativa com erro hipermetrópico. Já um procedimento refrativo corneano não resolveria o bloqueio capsular e também levaria a insatisfação refrativa com erro hipermetrópico, caso o bloqueio sofresse resolução espontânea mais tardiamente ou após capsulotomia por catarata secundária. Diante do exposto, sugerimos que em todo caso de suspeita de erro biométrico com desvio miópico seja considerada a possibilidade de síndrome do bloqueio capsular precoce. Para realizar o diagnóstico diferencial entre as duas situações, sugerimos o estudo cuidadoso da relação entre a lente e o saco capsular, sempre realizado sob midríase farmacológica.

\section{Abstract}

We present a case of early capsular block syndrome that was treated by re-operation on the second post operative day. We discuss the major characteristics of the syndrome, possible predisposing factors, therapeutic options and highlight the importance of its correct recognition. We also speculate about another possible risk factor, an intraocular lens with a optic part about $6 \mathrm{~mm}$ or more.

Keywords: Lens capsule; Lens/surgery; Cataract extraction/adverse effects; Pseudophakia/complications; Case reports

\section{REFERÊNCIAS}

1. Davison JA. Capsular bag distension after endophacoemulsification and posterior chamber intraocular lens implantation. J Cataract Refract Surg. 1990; 16(1):99-108.

2. Miyake K, Ota I, Ichihashi S, Miyake S, Tanaka Y, Terasaki H. New classification of capsular block syndrome. J Cataract Refract Surg. 1998; 24(9):1230-4.

3. Kim HK, Shin JP. Capsular block syndrome after cataract surgery: clinical analysis and classification. J Cataract Refract Surg. 2008; 34(3):357-63.

4. Durak I, Ozbek Z, Ferliel ST, Oner FH, Soylev M. Early postoperative capsular block syndrome. J Cataract Refract Surg. 2001; 27(4):555-9.

5. Mardelli PG. Slitlamp needle revision of capsular block syndrome. J Cataract Refract Surg. 2008; 34(7):1065-9.

6. Marback EF. Resultados iniciais com a técnica de facoemulsificação por múltipla divisão circunferencial. Rev Bras Oftalmol. 2005;64:87-91.

7. Masket S. Postoperative complications of capsulorhexis. J Cataract Refract Surg. 1993; 19(6):721-4.

8. Sugiura T, Miyauchi S, Eguchi S, Obata H, Nanba H, Fujino Y, et al. Analysis of liquid accumulated in the distended capsular bag in early postoperative capsular block syndrome. J Cataract Refract Surg. 2000; 26(3):420-5.

9. Mardelli PG, Mehanna CJ. Phacoanaphylactic endophthalmitis secondary to capsular block syndrome. J Cataract Refract Surg. 2007; 33(5):921-2.

\section{Endereço para correspondência: \\ Eduardo Marback \\ R. Jequié, $n^{\circ} 4$ - Rio Vermelho \\ CEP 41940-580 - Salvador - Bahia \\ Tel: (71) 3330-6100}

Fax: (71) 3330-6114 